\title{
Coherent control of bound entangled electrons in a CdMnTe quantum well
}

\author{
P. W. Jacobs ${ }^{1}{ }^{\star}$, J. K. Furdyna ${ }^{2}$, and R. Merlin ${ }^{1}$ \\ ${ }^{1}$ University of Michigan, Ann Arbor, MI 48109, USA \\ ${ }^{2}$ University of Notre Dame, Notre Dame, IN 46556, USA
}

Received 10 November 2007, revised 1 April 2008, accepted 4 April 2008 Published online 17 June 2008

PACS 75.50.Pp, 75.70.Ak, 78.20.Ls, 78.47.J-, 78.67.De

* Corresponding author: e-mail jacobsp@umich.edu

Ultrafast laser pulses were used to control the spin state of a pair of entangled, donor-bound electrons in a CdMnTe quantum well. The entanglement, mediated by an optically-excited exciton, was characterized by pumpprobe spectroscopy. The data exhibits two dominant oscillations, one at the frequency of the spin-flip of a donor

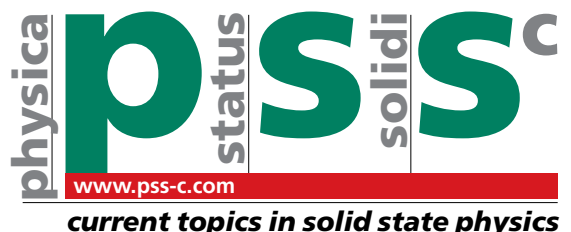

current topics in solid state physics electron and the second one at twice this frequency. Their amplitudes depend linearly on the pump power. The quantum state of the two-electron spin system can be controlled by appropriate spatial and temporal shaping of the pump pulses.

๑) 2008 WILEY-VCH Verlag GmbH \& Co. KGaA, Weinheim

1 Introduction Entangled states in semiconductors have been intensively studied in the last few years because of possible applications in quantum information science. Such systems combine the possibility of quantum computation with the scalability and advanced techniques of semiconductor processing. Examples include the spin degrees of freedom of donors and acceptors [1], and excitons in quantum dots [2]. In an earlier study, Bao et al. [3,4] reported on the exciton-mediated spin entanglement of up to three bound electrons in a CdMnTe quantum well. The exciton and the resulting entanglement were created and probed by ultrafast laser pulses. In recent years, tailored ultrafast optical pulses have been applied to a wide variety of systems to control vibrations [5], chemical reactions [6], and electronic excitations [7]. Here, we use shaped pulses to control the spin quantum state of an ensemble of electron pairs bound to nearby donors in the same system studied previously by Bao et al. [3,4].

2 Experiment We performed pump-probe differential magnetic Kerr measurements in the Voigt geometry using a Ti-sapphire laser (Tsunami, Spectra Physics) which provided $80 \mathrm{fs}$ pulses centered at $730 \mathrm{~nm}$. The signal of interest is the pump-induced shift of the polarization an- gle of the reflected probe. Both pump and probe beams were chopped by acousto-optic modulators, and a lock-in amplifier was used to detect at the difference frequency. The pump was circularly polarized to preferentially excite a particular spin orientation of the heavy hole. The incident probe was linearly polarized and its scattered polarization was measured using a polarizing beam splitter and a balanced Si photodetector, following the work of Crooker et al. [8]. The time-domain traces were analyzed in terms of a set of exponentially-decaying sinusoids using linear prediction methods [9]. We used a double array of liquid crystal pixels (Spatial Light Modulator, Cambridge Research and Instrumentation) to produce a variety of pulse shapes. The pulse shaper uses a grating to disperse the pulses' spectrum and a lens to collimate the diverging spectral elements along an axis perpendicular to the optical axis. The amplitude and phase of the spectral elements are controlled by two LCD arrays positioned between two polarizers [10], followed by a second lens that refocuses the beam onto a second grating that reassembles the pulses. The sample was placed in a magnetic cryostat and immersed in liquid He. Our material system, the same one used by Bao et al. [3,4], consists of 100 layers of 58 - $\AA$-thick $\mathrm{Cd}_{0.996} \mathrm{Mn}_{0.004} \mathrm{Te}$ sandwiched between 

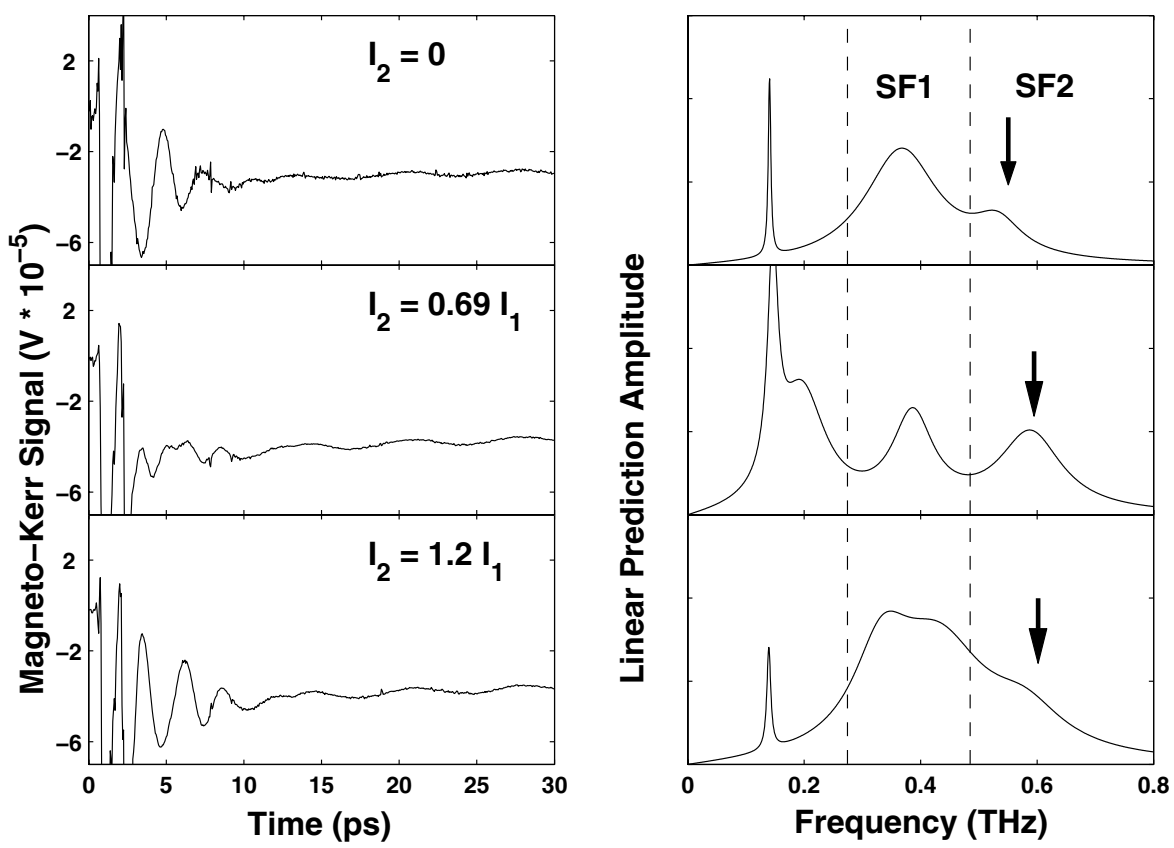

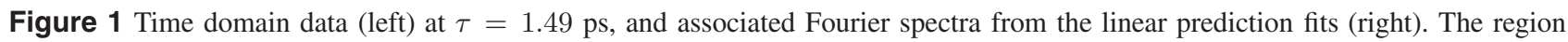
between the vertical dashed bars indicates the single electron spin flip transition. The overtone is indicated by arrows.

large bandgap antiferromagnetic 19- $\AA$-thick MnTe barriers. Although their concentration is rather small, the Mn ions lead to a large enhancement of the electron $g$-factor in the wells [11]. The quantum entanglement discussed here involves the spin component of the wavefunction of a pair of electrons bound to donors, tentatively identified as In. Their estimated concentration is $5 \times 10^{16} \mathrm{~cm}^{-3}[3,4]$.

3 Results and discussion Initially, the state of the bound electron spins at low temperatures is aligned along the external magnetic field. The work of Bao et al. $[3,4]$ showed that laser pulses in resonance with bound excitons in the CdMnTe layers create (coherent) quantum superposition states involving the $S=N / 2$ spin manifold, where $S$ is the total spin quantum number of a system of $N$ electrons; the corresponding two-electron states are the triplet $|\downarrow \downarrow\rangle,\{|\downarrow \uparrow\rangle+|\uparrow \downarrow\rangle\} / \sqrt{2}$ and $|\uparrow \uparrow\rangle$. This laserinduced entanglement relies on the fact that, at weak fields, the spin of the heavy-hole composing the exciton is perpendicular to the plane of the quantum well [12]. If the externally applied field is oriented in the plane, the exciton introduces through exchange coupling an effective shift in the direction of the net magnetic field felt by the donorbound electrons $[3,4]$. Since the quantization axes with and without the exciton are not the same, it is then possible to optically excite Raman transitions involving multiple spin flips and, thus, create many-spin Raman coherences [3,4]. Shaped laser pulses can control the relative intensity of the
Raman transitions and, thus, leave the spin system in a (to some degree) predetermined quantum state. Here, we show how the final quantum state is affected by a pair of pulses of varying intensity and separated by a controllable time delay.

Assume that, initially, the two-electron system is in the $S_{z}=-1$ state

$$
\psi(0)=|\downarrow \downarrow\rangle .
$$

Then, after interacting with the exciton created by the first pump pulse, the state becomes

$$
\begin{aligned}
\psi(t) & =C_{0}|\downarrow \downarrow\rangle+C_{1} e^{i E_{S F} t / \hbar}\{|\downarrow \uparrow\rangle \\
& +|\uparrow \downarrow\rangle / \sqrt{2}+C_{2} e^{i 2 E_{S F} t / \hbar}|\uparrow \uparrow\rangle
\end{aligned}
$$

where $E_{S F}$ is the single-electron spin-flip energy. At small laser intensities, $\left|C_{1}\right|,\left|C_{2}\right|<<1$ and $C_{0} \approx 1$. Therefore, the interaction with a second identical pulse results approximately in the state

$$
\begin{aligned}
\psi(t) \approx & C_{0}|\downarrow \downarrow\rangle \\
& +C_{1} e^{i E_{S F} t / \hbar}\left(1+e^{i E_{S F} \tau / \hbar}\right)\{|\downarrow \uparrow\rangle+|\uparrow \downarrow\rangle\} / \sqrt{2} \\
& +C_{2} e^{i 2 E_{S F} t / \hbar}\left(1+e^{i 2 E_{S F} \tau / \hbar}\right)|\uparrow \uparrow\rangle
\end{aligned}
$$

where $\tau$ is the time delay between the two pulses. It follows that the contribution of the $S_{z}=0\left(S_{z}=1\right)$ component of the triplet can be eliminated if the delay between the pulses is equal to a half (a quarter) of the spin-flip period. 


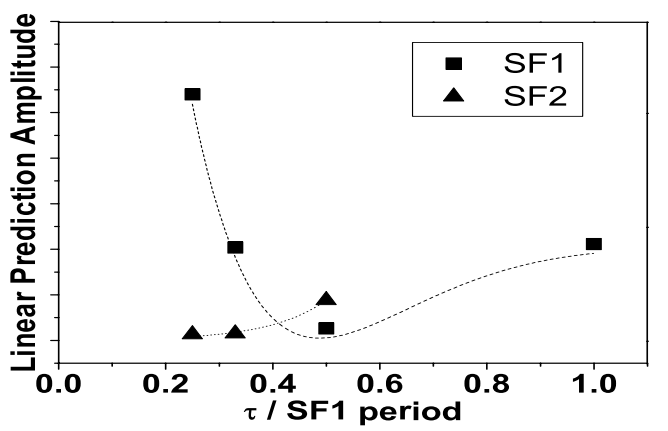

Figure 2 Amplitude of SF1 and SF2 as a function of normalized time separation between the pump pulses.

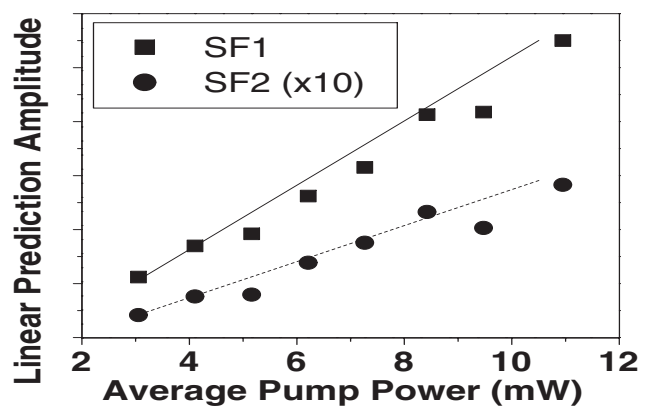

Figure 3 Dependence of SF1 and SF2 amplitudes on the pump laser power $\left(I_{2}=0\right)$.

However, this ignores the effects of decay, which are important here because the apparent lifetime of the spin flip oscillations is comparable to their period (the broadening is mainly of inhomogeneous nature). Thus, the second of the two pulses must be weaker to avoid overcompensating. To select the optimal relative intensity, we use the fact that the pulse shaper introduces not only a temporal delay, but also a spatial shift between the pulses so that the ratio of the pump intensities can be varied by simply moving the smaller probe [10].

Figure 1 shows the results of ultrafast pump-probe measurements using two pulses at the magnetic field $B=$ 5.25T. Here, $\tau$ was set to $1.49 \mathrm{ps,} \mathrm{which} \mathrm{is} \mathrm{approximately}$ half the period of the $S_{z}=-1 \rightarrow S_{z}=0$ oscillation. The time domain data was obtained by probing different regions of the overlapping pump spots. The intensity ratio of the two pump pulses at the probe spot is given in the figure. The first and second pump pulses arrive at 0.5 ps and 2.0 ps, and have identical intensities at their spatially-displaced peak locations. In the Fourier spectra from linear prediction fits, the sharp peak at $\sim 140 \mathrm{GHz}$ is the long-lived spin-flip oscillation from the Mn electrons [13]. The band labeled SF1 represents the short lived signal from the spin flip of a single donor bound electron $\left(S_{z}=-1 \rightarrow S_{z}=0\right)$.
The structure reflects both ground $(\sim 380 \mathrm{GHz})$ and excited state $(\sim 400 \mathrm{GHz})$ coherences $[3,4]$. Finally, SF2 at $\sim 590 \mathrm{GHz}$ is the simultaneous spin flip of a pair of donor bound electrons $\left(S_{z}=-1 \rightarrow S_{z}=1\right)$. The spectrum with a single pump pulse $\left(I_{2}=0\right)$ is dominated by the single electron spin flip. When the ratio between the two pump intensities is optimal, $I_{2}=0.69 I_{1}$, the amplitude of the ground state SF1 is reduced. The remaining peak is an excited state spin-flip coherence. When $I_{2}=1.2 I_{1}$, the first pulse is weaker than the second one and, due to decay, the spectrum is dominated by oscillations resulting from the second pulse alone. Following the method of [3], we can provide a quantitative measurement of the entanglement by using the ratio between the SF2 and SF1 amplitudes. From top to bottom, these ratios are $0.049,1.66$, and 0.58 . These numbers should be compared with the earlier, single-pulse result of 0.014 [3].

Figure 2 demonstrates that the reduction in intensity of the spin flip mode depends on $\tau$ in a manner consistent with Eq. (3). Here, the probe pulse has been set to the position corresponding to the optimal pump intensity ratio. The spin flip intensity exhibits a minimum when $\tau$ approaches half the period of SF1 before increasing again when $\tau$ reaches the period of the spin flip. The amplitude of SF1 does not return to its original value due to its rapid decay. Similarly, the amplitude of SF2 increases as $\tau$ nears the period of $\mathrm{SF} 2$, but the signal decays too rapidly for it to be observed when $\tau$ equals the SF1 period. Finally, Fig. 3 shows that both the SF1 and SF2 amplitudes depend linearly on the average (single) pump power, excluding the possibility that the second harmonic signal is due to second-order scattering of the probe.

4 Conclusions We have demonstrated coherent control of two entangled electrons in a dilute magnetic semiconductor quantum well using a pair of ultrafast laser pulses. The effects of the separation between the pulses and their relative intensities on the entanglement have been demonstrated. The linear nature of the scattering process has also been studied, and the results support the model described in $[3,4]$.

Acknowledgements This work was supported by the NSF Focus Physics Frontier Center, the NSF Grant DMR 0603752 and by the DARPA SpinS Program.

\section{References}

[1] B. E. Kane, Nature 393, 133 (1998).

[2] X. Li, Y. Wu, D. Steel, D. Gammon, T. H. Stievater, D. S. Katzer, D. Park, and C. Piermarocchi, Science 301, 809 (2003).

[3] J. M. Bao, A. V. Bragas, J. K. Furdyna, and R. Merlin, Phs. Rev. B 71, 045314 (2005).

[4] J. M. Bao, A. V. Bragas, J. K. Furdyna, and R. Merlin, Nat. Mater. 2, 175 (2003).

[5] U. Ozgur, C. Lee, and H. O. Everitt, Phys. Rev. Lett. 86, 5604 (2001). 
[6] E. C. Carroll, B. J. Pearson, A. C. Florean, P. H. Bucksbaum, and R. J. Sension, J. Chem. Phys. 124, 114506 (2006).

[7] A. P. Heberle, J. J. Baumberg, and K. Köhler, Phys. Rev. Lett. 75, 2598 (1995).

[8] S. A. Crooker, D. D. Awschalom, and N. Samarth, IEEE J. Select. Top. Quantum Electron. 1, 1082 (1995).

[9] H. Barkhuijsen, R. de Beer, W. M. M. J. Bovée, and D. van Ormondt, J. Mag. Res. 61, 465 (1984).

[10] M. Wefers and K. A. Nelson, J. Opt. Soc. Am. B 12, 1343 (1995).

[11] J. K. Furdyna, J. Appl. Phys. 64, R29 (1988).

[12] P. Peyla, A. Wasiela, Y. M. d'Aubigné, D. E. Ashenford, and B. Lunn, Phys. Rev. B 47, 3783 (1993).

[13] J. Stühler, G. Schaack, M. Dahl, A. Waag, G. Landwehr, K. V. Kavokin, and I. A. Merkulov, Phys. Rev. Lett. 74, 2567 (1994). 